\title{
Cloning, expression and rapid purification of active recombinant mycothiol ligase as B1 immunoglobulin binding domain of streptococcal protein $\mathrm{G}$, glutathione- $S$-transferase and maltose binding protein fusion proteins in Mycobacterium smegmatis
}

\author{
Maria-Teresa Gutierrez-Lugo ${ }^{\text {a }}$, Gerald L. Newton ${ }^{\mathrm{b}}$, Robert C. Fahey ${ }^{\mathrm{b}}$, Carole A. Bewley ${ }^{\mathrm{a}, *}$ \\ ${ }^{a}$ Laboratory of Bioorganic Chemistry, National Institute of Diabetes and Digestive and Kidney Diseases, National Institutes of Health, \\ 9000 Rockville Pike, Bldg 8, Room 1A-02, Bethesda, MD 20892-0820, USA \\ ${ }^{\mathrm{b}}$ Department of Chemistry and Biochemistry, University of California, San Diego, La Jolla, CA 92093, USA
}

Received 11 April 2006, and in revised form 22 June 2006

Available online 11 July 2006

\begin{abstract}
Mycothiol ligase (MshC) is a key enzyme in the biosynthesis of mycothiol, a small molecular weight thiol found in Mycobacteria spp. and other actinomycetes. Mycothiol plays a fundamental role in these organisms by helping to provide protection from the effects of reactive oxygen species and electrophiles, including many antibiotics. It has recently been demonstrated that the MshC gene and more generally the production of mycothiol are essential to Mycobacterium tuberculosis, indicating that MshC may represent a novel target for new classes of antituberculars. Because MshC cannot be expressed heterologously in Escherichia coli and isolation from Mycobacterium smegmatis is impractical, we have optimized the E. coli-M. smegmatis shuttle vector pACE for cloning and recombinant expression of MshC (under control of an acetamidase-inducible promoter). To improve expression levels and simplify purification, we further constructed three N-terminal-MshC fusion proteins where N-terminal tags included the B1 domain of streptococcal protein G (to give GB1$\mathrm{MshC}$ ), glutathione-S-transferase (to give GST-MshC) and maltose binding protein (to give MBP-MshC), for expression in $M$. smegmatis. By expressing all three fusion proteins in a mutant strain of $M$. smegmatis mc $^{2} 155$, namely I64 L205P MshC M. smegmatis which lacks mycothiol ligase activity, we demonstrate in vivo mycothiol ligase activity for each construct. Recombinant GST-MshC and MBP-MshC were isolated in one step by affinity chromatography in a yield of 0.7 and $1.2 \mathrm{mg}$ fusion protein/L and exhibited specific activities of

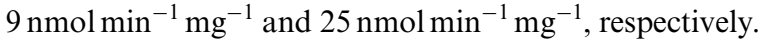

(C) 2006 Elsevier Inc. All rights reserved.

Keywords: MshC; Mycothiol; Fusion proteins; Affinity purification; Mycothiol ligase; Complementation; Mycobacterium tuberculosis; Shuttle vector

\section{Introduction}

Mycothiol (MSH, AcCys-GlcN-Ins) ${ }^{1}$ is the dominant low molecular weight thiol that is so far unique to actinomycetes
$[1,2]$ and has been shown to be present in all major groups of actinomycetes [1]. Mycothiol comprises one unit each of $\mathrm{N}$-acetylcysteine (AcCys), 1-D-myo-inositol (Ins) and 2amino-2-deoxy-D-glucopyranose (GlcN). Mycothiol is

\footnotetext{
* Corresponding author. Fax: +1 3014020008.

E-mail address: caroleb@mail.nih.gov (C.A. Bewley).

1 Abbreviations used: MSH, AcCys-GlcN-Ins, 1-D-myo-inosityl-2-( $N$-acetyl-L-cysteinyl) amido-2-deoxy- $\alpha$-D-glucopyranoside; GlcN-Ins, 1-D-myo-inosityl-2-amino-2-deoxy- $\alpha$-D-glucopyrano-side; Cys-GlcN-Ins, 1-D-myo-inosityl-2-(L-cysteinyl) amido-2-deoxy-D-glucopyranoside; SDS-PAGE, sodium dodecyl sulfate-polyacrylamide gel electrophoresis; GB1, B1 immunoglobulin binding domain of streptococcal protein G; GST, glutathione-S-transferase; MBP, maltose binding protein; DTT, dithiothreitol; SDM, site directed mutagenesis; DAD, diode array detector; API-ES, atmospheric pressure electrospray ionization; MSD, mass selective detector; ORF, open reading frame.
} 


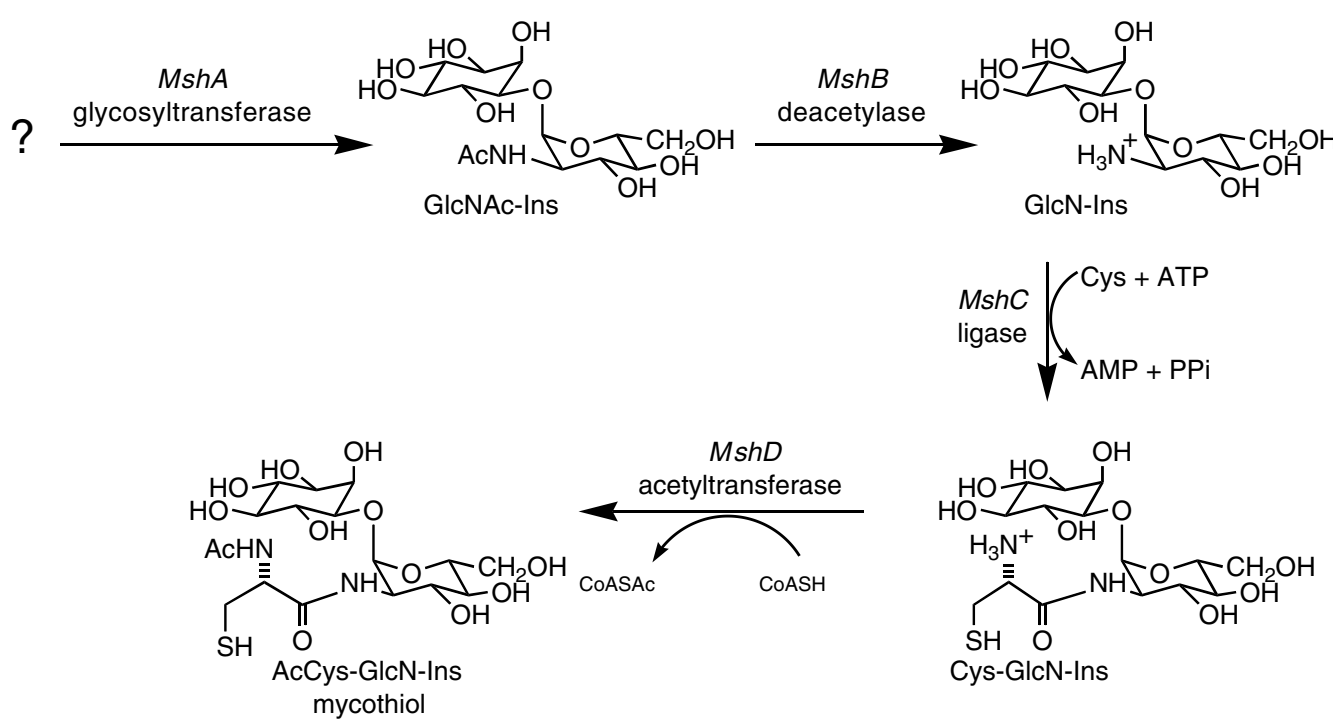

Fig. 1. Biosynthetic scheme of mycothiol.

assumed to be the functional equivalent of glutathione in mycobacteria and has been shown to be involved in protecting Mycobacterium tuberculosis and Mycobacterium smegmatis from toxic oxidants and antibiotics [3,4]. Owing to the demonstration that mycothiol-deficient mutants become hypersensitive to some first line antituberculars, interest in identifying inhibitors of mycothiol biosynthesis and mycothiol-assisted detoxification has continued to rise [5].

Mycothiol biosynthesis occurs in at least four steps and involves the gene products of $m s h A$ through $m s h D$. The third committed step in mycothiol biosynthesis involves transfer of L-cysteine (Cys) to GlcN-Ins to produce CysGlcN-Ins and is catalyzed by the enzyme mycothiol ligase, or $m s h C$ (L-Cys:1D-myo-inosityl 2-amino-2-deoxy- $\alpha$-Dglucopyranoside ligase), in an ATP-dependent manner $[2,6]$ (Fig. 1). Recently, the $m s h C$ gene has been shown to be essential for growth in $M$. tuberculosis by targeted gene disruption [7] and by transposon site hybridization [8]. When a second copy of $m s h C$ was inserted into the genome prior to targeted inactivation of the native $m s h C$ gene, viable mutants were isolated [7]. Since MshC has been shown to be required for MSH biosynthesis [4], these studies indicate that mycothiol is essential for growth in M. tuberculosis strains provoking further study of this enzyme as a therapeutic target.

The initial report describing the identification and characterization of MshC relied on laborious isolation methods from large scale cultures of $M$. smegmatis that were further hampered by the production of two MshC protein products that differed by addition of eight amino acids on the N-terminus [6]. In contrast to heterologous production of most proteins that can be expressed well in Escherichia coli, there is ample evidence that the proteins derived from $M$. tuberculosis are refractory to these conditions [9-12]. Because a prerequisite for screening small molecule libraries to identify inhibitors of MshC will rely on reproducible production of relatively large amounts of active enzyme, we have optimized the E. coli-mycobacterial shuttle vector pACE $[13,14]$ to provide a robust cloning and expression vector system for production of MshC using M. smegmatis as the expression host. Fusion to three unrelated proteins through the N-terminus of MshC increases production and greatly facilitates purification. We believe these expression vectors will be of general value for production of proteins derived from M. tuberculosis.

\section{Materials and methods}

Construction of mshC-tagged expression plasmids $p A C E /$ GBI-MshC, $p A C E / G S T-M \operatorname{sh} C$ and $p A C E / M B P-M s h C$

The MshC gene was previously amplified from total genomic DNA of $M$. tuberculosis strain $\mathrm{H} 37 \mathrm{Rv}$ and cloned into the BamHI/HindIII sites of pRSETA (Invitrogen) [6]. The resulting plasmid pRSETA/MshC was used as a template for PCR amplification of MshC using Taq PCR master mix kit according to the manufacturer's instructions (Qiagen) together with an appropriate pair of forward and reverse primers designed for each of three fusion protein constructs. The primer sequences used are shown in Table 1 and numbered 1, 2 and 4 . The amplified MshC gene products were subcloned into the BamHI/XhoI sites of pGEV2 [15], NcoI/ BamHI of pET-42a (Novagen) or BamHI/HindIII sites of pMALc2X (New England Biolabs) using a quick ligation kit (New England Biolabs) to generate constructs encoding GB1, GST- $\mathrm{H}_{6}-\mathrm{S}$ and MBP fusion proteins, respectively. Note that insertion of the MshC gene into the $N c o \mathrm{I} /$ BamHI sites of pET-42a generates a fusion construct in which GST and $\mathrm{MshC}$ are separated by a 6-His and $\mathrm{S}$ tag-linker, referred to above as GST- $\mathrm{H}_{6}-\mathrm{S}$ and as GST from here on. The corresponding constructs, referred to as $\mathrm{pGEV} / \mathrm{MshC}$ (for expression of GB1-MshC), pET42a/MshC-2 (for expression of GST-MshC after frame correction of pET42a/MshC by primer set 3 listed in Table 1) and pMALc2X/MshC (for expression of MBP-MshC), were subsequently used as tem- 
Table 1

Summary of primers and templates used in this study and plasmids generated

\begin{tabular}{|c|c|c|c|c|c|}
\hline Primer set & Primer ID & Primer sequence ${ }^{\mathrm{a}, \mathrm{b}}$ & Restriction site & Template & Construct \\
\hline 1 & $\begin{array}{l}\text { 5'GEV-MshC } \\
\text { 3'GEV-MshC }\end{array}$ & $\begin{array}{l}5^{\prime} \text {-gcttatGGATCCatgcagtcgtgg-3' } \\
5^{\prime} \text {-agttcaCTCGAGctacaggtccacc-3' }\end{array}$ & $\begin{array}{l}\text { BamHI } \\
\text { XhoI }\end{array}$ & pRSETA/MshC & $\mathrm{pGEV} / \mathrm{MshC}$ \\
\hline 2 & $\begin{array}{l}\text { 5'42a-MshC } \\
\text { 3'42a-MshC }\end{array}$ & $\begin{array}{l}5^{\prime} \text {-gcttatCCATGGatgcagtcgtgg- } 3^{\prime} \\
5^{\prime} \text {-agttcaGGATCCctacaggtccacc-3' }\end{array}$ & $\begin{array}{l}\text { NcoI } \\
\text { Bam HI }\end{array}$ & pRSETA/MshC & $\mathrm{pET} 42 \mathrm{a} / \mathrm{MshC}$ \\
\hline 3 & $\begin{array}{l}5^{\prime} 42 \mathrm{aX}-\mathrm{MshC} \\
3^{\prime} 42 \mathrm{aX}-\mathrm{MshC}^{\mathrm{c}}\end{array}$ & $\begin{array}{l}5^{\prime} \text {-ggtattgagggacgcgggccatggatgcagtcgtgg-3' } \\
5^{\prime} \text {-ccacgactgcatccatggcccgcgtccctcaatacc-3' }\end{array}$ & $\begin{array}{l}\text { N/A* } \\
\text { N/A* }\end{array}$ & $\mathrm{pET} 42 \mathrm{a} / \mathrm{MshC}$ & pET42a/MshC-2 \\
\hline 4 & $\begin{array}{l}\text { 5'GEV-MshC } \\
\text { 3'MBP-MshC }\end{array}$ & $\begin{array}{l}5^{\prime} \text {-gcttatGGATCCatgcagtcgtgg-3' } \\
5^{\prime} \text {-agttcaAAGCTTctacaggtccacc-3' }\end{array}$ & $\begin{array}{l}\text { BamHI } \\
\text { HindIII }\end{array}$ & pRSETA/MshC & pMALc2x/MshC \\
\hline 5 & $\begin{array}{l}\text { 5'pACEMshC-1 } \\
\text { 3'pACEMshC-1 }\end{array}$ & $\begin{array}{l}5^{\prime} \text {-gataagagaaagggagtc cacatatgggatccatgcagtcgtgg- } 3^{\prime} \\
5^{\prime} \text {-ccacgactgcatggatcccatatgtggactccctttctcttatc- } 3^{\prime}\end{array}$ & $N d e \mathbf{I}$ & $\mathrm{pACE} / \mathrm{MshC}$ & $\mathrm{pACE} / \mathrm{MshC}-1$ \\
\hline 6 & $\begin{array}{l}\text { 5'pACEMshC-2 } \\
\text { 3'pACEMshC-2 }\end{array}$ & $\begin{array}{l}5^{\prime} \text {-gaaaggcagtccacatatgactagtggatccatgcagtcgtgg- } 3^{\prime} \\
5^{\prime} \text {-ccacgactgcatggatccactagtcatatgtggactccctttc- } 3^{\prime}\end{array}$ & SpeI & $\mathrm{pACE} / \mathrm{MshC}-1$ & $\mathrm{pACE} / \mathrm{MshC}-2$ \\
\hline 7 & $\begin{array}{l}5^{\prime} \mathrm{GB} 1 \\
3^{\prime} \mathrm{GB} 1\end{array}$ & $\begin{array}{l}5^{\prime} \text {-gtcaggCATATGcagtacaagctt- } 3^{\prime} \\
5^{\prime} \text {-tacacgGGATCCacgcggaaccag- } 3^{\prime}\end{array}$ & $\begin{array}{l}\text { NdeI } \\
\text { Bam HI }\end{array}$ & pGEV & $\mathrm{pACE} / \mathrm{GB} 1-\mathrm{MshC}$ \\
\hline 8 & $\begin{array}{l}\text { 5'GST-MshCf } \\
\text { 3'GST-MshCf }\end{array}$ & $\begin{array}{l}5^{\prime} \text {-aagcgcggatcccatatgtcccct- } 3^{\prime} \\
5^{\prime} \text {-tgctgtAACGTTctacaggtccacccegagcag- } 3^{\prime}\end{array}$ & $\begin{array}{l}N d e \mathrm{I} \\
A c l \mathrm{I}\end{array}$ & pET42a/MshC-2 & $\mathrm{pACE} / \mathrm{GST}-\mathrm{MshC}$ \\
\hline 9 & $\begin{array}{l}\text { 5'MBP } \\
\text { 3'GST-MshCf }\end{array}$ & $\begin{array}{l}5^{\prime} \text {-cagcgaCATATGaaaatcgaagaa- } 3^{\prime} \\
5^{\prime} \text {-tgctgtAACGTTctacaggtccaccccgagcag- } 3^{\prime}\end{array}$ & $\begin{array}{l}N d e \mathrm{I} \\
\text { AclI }\end{array}$ & pMALc2X/MshC & $\mathrm{pACE} / \mathrm{MBP}-\mathrm{MshC}$ \\
\hline
\end{tabular}

a Capital letters indicate the added restriction sites in the PCR amplification.

b Underlined letters indicate the added restriction sites by site directed mutagenesis.

c Primers were designed to delete a single nucleotide located $5^{\prime}$ to the $N c o$ I restriction site.

* Not applicable.

plates for PCR amplification of individual tags and/or the complete fusion protein sequences for cloning into the pACE shuttle vector (Table 1) [13,14].

Prior to insertion of genes encoding tags or complete fusion proteins into the vector, additional cloning sites $N d e \mathrm{I}$ and SpeI were introduced into pACE/MshC by site directed mutagenesis (Stratagene) using the primer sets 5 and 6 to produce $\mathrm{pACE} / \mathrm{MshC}-1$ and $\mathrm{pACE} / \mathrm{MshC}-2$, respectively. Separate plasmid preparations of pACE/MshC-2 were digested with $\mathrm{Nde} \mathrm{I} / \mathrm{ClaI}$ or $\mathrm{Nde} / \mathrm{Bam} \mathrm{H} 1$ and dephosphorylated with Antarctic phosphatase (NEB) for cloning of NdeI/ AclI digested GST-MshC and MBP-MshC fragments, amplified from $\mathrm{pET} 42 \mathrm{a} / \mathrm{MshC}-2$ and $\mathrm{pMALc} 2 \mathrm{x} / \mathrm{MshC}$ using the set of primers 8 and 9 , respectively; or a $N d e \mathrm{I} / \mathrm{Bam} \mathrm{HI}$ digested GB1 fragment amplified from pGEV2 using primer set 7 (Table 1). Ligation of these fragments into complementary linear pACE/MshC-2 was performed using T4 DNA ligase (NEB) at $16^{\circ} \mathrm{C}$ overnight. The ligation mixtures were purified by ethanol precipitation [16] and electroporated into Electromax E. coli DH10B (Invitrogen) according to the manufacturer's instructions. The constructs, identified as pACE/GB1MshC, pACE/GSTMshC and pACE/MBPMshC, were verified by restriction analysis and DNA sequencing (Fig. 2). All sequences have been deposited in GenBank.

\section{Transformation and isolation of recombinant I64 M. smegmatis strains}

The different expression plasmids were electroporated into the I64 L205P MshC M. smegmatis mutant (referred to as
I64 M. smegmatis) that is devoid of cysteine ligase activity and therefore mycothiol production [4]. Preparation of I64 $M$. smegmatis competent cells and electroporation procedures were performed following published protocols [17]. Briefly, I64 M. smegmatis was grown in 7H9/OADC/Tween (comprising Middlebrook $7 \mathrm{H} 9$ (DIFCO) media supplemented with $10 \%$ OADC (Oleic acid, albumin, dextrose, catalase, BBL) and $0.05 \%$ Tween 20) until midlog phase. After harvesting, cells were washed twice with cold $10 \%$ glycerol by resuspension and centrifugation. I64 M. smegmatis competent cells in $10 \%$ cold glycerol $(0.06 \mathrm{ml})$ were transformed by electroporation in the presence of $1 \mu \mathrm{g}$ of DNA $(1.2 \mathrm{kV})$ in prechilled $1 \mathrm{~mm}$ gap cuvettes using a Multiporator ${ }^{\circledR} /$ Electroporator 2510 (Eppendorf, Hamburg, Germany). Cells were harvested with $1 \mathrm{ml}$ of warm 7H9/OADC/Tween and incubated for $2 \mathrm{~h}$ at $37^{\circ} \mathrm{C}$ with shaking $(225 \mathrm{rpm})$. An aliquot $(25 \mu \mathrm{l})$ of this culture was plated on 7H9/OADC/Tween agar plates containing $75 \mu \mathrm{g} / \mathrm{ml}$ hygromycin (Invitrogen). Plates were incubated at $37^{\circ} \mathrm{C}$ for three days to obtain the transformed recombinant strains.

\section{Expression of recombinant GB1-MshC, GST-MshC and $M B P-M$ shC fusion proteins}

The selected I64 M. smegmatis recombinant strains were grown in $25 \mathrm{ml} 7 \mathrm{H} 9 / \mathrm{OADC} / \mathrm{T}$ ween containing $75 \mu \mathrm{g} /$ $\mathrm{ml}$ hygromycin for $72 \mathrm{~h}$ at $37^{\circ} \mathrm{C}$ with shaking $(225 \mathrm{rpm})$. A 1 -ml aliquot of this starter culture was transferred to $100 \mathrm{ml}$ of $7 \mathrm{H} 9$ containing $1 \%$ glucose, $0.05 \%$ Tween 20 and $75 \mu \mathrm{g} / \mathrm{ml}$ hygromycin and incubated overnight at 

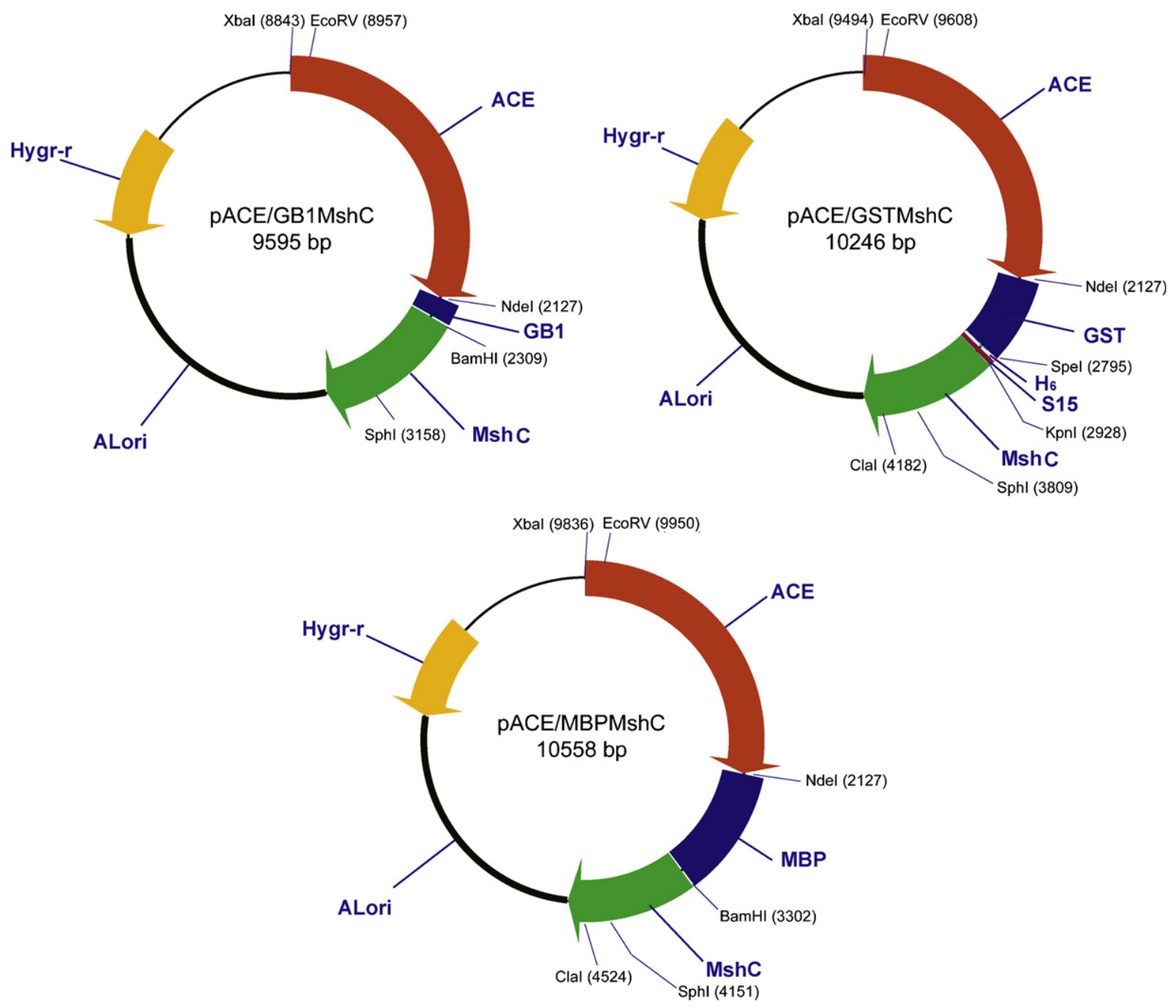

Fig. 2. Maps of mycobacterial expression vectors generated in this study encoding N-terminal-tagged MshC. Unique restriction sites are labeled and their locations indicated in parentheses. Proteins are labeled in bold and denoted by thick lines on the maps with the $\mathrm{N}$-terminal tag and MshC colored blue and green, respectively.

$37^{\circ} \mathrm{C}$ with shaking. After incubation, cells were harvested by centrifugation at $8000 \mathrm{~g}$ for $15 \mathrm{~min}, 4^{\circ} \mathrm{C}$ (Beckman Avanti J-20 XP centrifuge) and resuspended to an $\mathrm{A}_{600}$ of 0.3 in fresh $7 \mathrm{H} 9$ medium containing $0.4 \%$ acetamide (Sigma), $0.05 \%$ Tween 20 and $75 \mu \mathrm{g} / \mathrm{ml}$ hygromycin (referred to as expression medium) and incubated at $37^{\circ} \mathrm{C}$ with shaking (225 rpm). Cells were harvested after $22-26 \mathrm{~h}$ by centrifugation $\left(8000 \mathrm{~g}, 4^{\circ} \mathrm{C}\right.$ for $\left.15 \mathrm{~min}\right)$ and stored at $-80^{\circ} \mathrm{C}$ until further use.

Frozen cell pellets were thawed and resuspended to $25 \%$ $\mathrm{w} / \mathrm{v}$ cell suspension in cold lysis buffer containing $50 \mathrm{mM}$ HEPES, pH 7.5 (Cellgro), $3 \mathrm{mM} \quad \beta$-mercaptoethanol (Aldrich), $5 \mathrm{mM} \mathrm{MgCl}$ (Quality biological Inc.) and protease inhibitors (Complete, EDTA-free protease inhibitor cocktail tablets, Roche). The cells were disrupted by ultrasonication in an ice bath using an Ultrasonic Processor XL sonicator (Misonix Inc.) for 7 cycles ( $3 \mathrm{~s}$ on, $3 \mathrm{~min}$ off) each. Cell lysates were centrifuged at $3000 \mathrm{~g}$ for $1 \mathrm{~h}$ at $4{ }^{\circ} \mathrm{C}$, and supernatants were transferred to clean tubes. Cell lysates were analyzed by SDS-PAGE for protein expression (Phastgels ${ }^{\text {TM }}$ Gradient 10-15, Amersham Biosciences) and visualized by staining with Coomassie Blue (Coomassie $^{\mathrm{TM}}$ Blue R-350, Amersham Biosciences).

Detection of ligase activity by means of mycothiol production by recombinant $I 64 M$. smegmatis $p A C E / G B 1-M s h C$, pACE/GST-MshC and $p A C E / M B P-M$ shC strains

I64 M. smegmatis recombinant strains were grown in expression medium $(100 \mathrm{ml})$ under the conditions described above. The cultures were incubated for up to $55 \mathrm{~h}$ and aliquots of $1-12 \mathrm{ml}$ (depending on cell density) were taken during incubation to give cell pellets of approximately $10 \mathrm{mg}$ wet weight. Each aliquot was transferred to a tared $1.5 \mathrm{ml}$ microcentrifuge tube and cells pelleted at $12,100 \mathrm{~g}$ for $10 \mathrm{~min}$ at $4^{\circ} \mathrm{C}$ (Sorvall Biofuge Fresco); cell pellets were stored at $-20^{\circ} \mathrm{C}$ until further use.

Pellets were extracted and analyzed for total mycothiol content, and mycothiol production was quantified employing a modified method described previously by Newton et al. [1]. Briefly, cell pellets were lysed by vortexing in $160 \mu \mathrm{l}$ of reducing lysis buffer (50 mM HEPES 7.5 in $50 \%$ 
$\mathrm{CH}_{3} \mathrm{CN}: \mathrm{H}_{2} \mathrm{O}, 4 \mathrm{mM}$ DTT) followed by incubation at $60^{\circ} \mathrm{C}$ for $30 \mathrm{~min}$ with constant vortexing. Reduced thiols were derivatized by addition of $40 \mu \mathrm{l}$ of $100 \mathrm{mM}$ monobromobimane in acetonitrile and kept at $60^{\circ} \mathrm{C}$ for 10 min with occasional vortexing, and then placed on ice and quenched with $75 \mu \mathrm{l}$ of $0.5 \mathrm{M}$ methanesulfonic acid (Fluka). Samples were centrifuged at $12,100 \mathrm{~g}$ at $4{ }^{\circ} \mathrm{C}$ for $10 \mathrm{~min}$. Supernatants $(2 \mu \mathrm{l})$ were analyzed for total mycothiol content by LC-MS plus fluorescence detection using the following chromatographic conditions: Waters Symmetry C18 $5 \mu \mathrm{m}$ column $(4.6 \times 150 \mathrm{~mm})$ fitted with a QR $5 \mu \mathrm{m} \mathrm{C18}$ guard cartridge $(4.6 \mathrm{~mm} \times 1 \mathrm{~cm})$, gradient elution (flow rate of $0.5 \mathrm{ml} \mathrm{min}^{-1}$ ) of $5 \% \mathrm{MeOH} / 95 \% 0.15 \%$ formic acid in water to $100 \%$ $\mathrm{MeOH}$ in $15 \mathrm{~min}$ followed by a 2 -min wash with $100 \%$ $\mathrm{MeOH}$. The column was reequilibrated with $5 \% \mathrm{MeOH} /$ $95 \% 0.15 \%$ formic acid for 3 min prior to each injection. Detection of derivatized thiols was accomplished using a fluorescence detector (Agilent Technologies) with excitation at $398 \mathrm{~nm}$ and emission at $480 \mathrm{~nm}$. Under these chromatographic conditions mycothiol bimane eluted at $9.9 \mathrm{~min}$ $(\mathrm{m} / \mathrm{z}$ 677, 699, 715). Total mycothiol content $(\mu \mathrm{mol} / \mathrm{g}$ wet weight) in each aliquot was calculated by comparing peak areas against a mycothiol calibration curve.

\section{Purification and characterization of GST-MshC and MBP- MshC from recombinant I64 M. smegmatis strains}

To obtain recombinant GST-MshC or MBP-MshC fusion proteins, respective I64 M. smegmatis strains I64pACE/GST-MshC and I64pACE/MBP-MshC were grown in $4 \times 500 \mathrm{ml}$ ( $1 \mathrm{~L}$ flasks) of expression medium (described above) inoculated with $3-4 \mathrm{ml}$ each of a starter culture grown in $7 \mathrm{H} 9$ supplemented with $1 \%$ glucose, $0.05 \%$ Tween 20 and $75 \mu \mathrm{g} / \mathrm{ml}$ hygromycin. The cultures $\left(A_{600} 0.3\right)$ were incubated at $37^{\circ} \mathrm{C}(225 \mathrm{rpm})$ for $22-26 \mathrm{~h}$ and cell lysates were prepared by sonication at $4^{\circ} \mathrm{C}$ for 7 cycles $(3 \mathrm{~s}$ on, $3 \mathrm{~min}$ off) each, followed by centrifugation at $4^{\circ} \mathrm{C}$ for $1 \mathrm{~h}$ at $18,000 \mathrm{~g}$. All subsequent purification steps were carried out at $4{ }^{\circ} \mathrm{C}$.

The resulting protein extracts were diluted $1: 1 \mathrm{v} / \mathrm{v}$ with appropriate binding buffer before loading onto an affinity resin column equilibrated in the same buffer. For GST-MshC, supernatants were diluted with GST bind/wash buffer (Novagen) and loaded onto a column containing $12 \mathrm{ml}$ GST Sepharose ${ }^{\mathrm{TM}}$ affinity resin (Novagen, $1.6 \times 7.5 \mathrm{~cm}$ ). MBPMshC supernatants were diluted with $50 \mathrm{mM}$ HEPES, $200 \mathrm{mM} \mathrm{NaCl}, \mathrm{pH} 7.5$, and loaded onto a column containing $20 \mathrm{ml}$ amylose resin (New England Biolabs, $2.6 \times 5 \mathrm{~cm}$ ). Using an ÄKTAprime plus chromatographic system (GE Healthcare), columns were washed with 10 column volumes of binding buffer followed by elution with three column volumes of $10 \mathrm{mM}$ reduced glutathione (Novagen) in binding buffer for GST-MshC (flow rate $0.8 \mathrm{mlmin}^{-1}$ ) or $10 \mathrm{mM}$ maltose (Sigma) in $50 \mathrm{mM}$ HEPES, pH 7.5, $200 \mathrm{mM} \mathrm{NaCl}$ for MBPMshC (flow rate of $1 \mathrm{mlmin}^{-1}$ ). All binding and elution buffers also contained $5 \mathrm{mM} \mathrm{MgCl}_{2}, 3 \mathrm{mM} \quad \beta$-mercaptoethanol and $5 \mathrm{mM}$ benzamidine (Sigma) as protease inhibi- tor. Fractions $(2 \mathrm{ml})$ were collected during the elution step and analyzed by SDS-PAGE for detection of fusion proteins. Fractions containing the desired recombinant protein were pooled and stored as $20 \%$ glycerol stocks at $-80^{\circ} \mathrm{C}$. Protein concentrations were determined by $A_{280}$ measurements and calculated using theoretical extinction coefficients of $127,825 \mathrm{M}^{-1} \mathrm{~cm}^{-1}$ for GST-MshC and $145,565 \mathrm{M}^{-1} \mathrm{~cm}^{-1}$ for MBP-MshC (http://us.expasy.org/tools/protparam.html) [18].

Identity of the purified recombinant proteins was confirmed by mass spectrometry analysis using an Agilent Technologies LCMS equipped with DAD detector and API-ES ionization source. Proteins were eluted from a C3 reverse phase column (Zorbax 300SB-C3, $2.1 \times 150 \mathrm{~mm}$, $5 \mu \mathrm{m}$, Agilent) using a linear gradient of $5-95 \%$ acetonitrile in $5 \%$ acetic acid over $25 \mathrm{~min}$ at a flow rate of $0.2 \mathrm{ml} \mathrm{min}^{-1}$ at $40^{\circ} \mathrm{C}$, and detected by UV at $280 \mathrm{~nm}$ and positive APIES. The retention times for GST-MshC and MBP-MshC under these conditions were 41.1 and $40.5 \mathrm{~min}$, respectively. Deconvolution of the mass spectra indicated molecular weights of $77.28 \mathrm{kDa}$ and $88.68 \mathrm{kDa}$, in excellent agreement with the predicted masses of 77.2758 and $88.6791 \mathrm{kDa}$.

\section{Detection of mycothiol ligase activity}

Mycothiol ligase activity of the I64 M. smegmatis transformed strains was measured by a modified method reported by Sareen et al. [6]. Enzyme activity was assayed in $0.2 \mathrm{ml}$ micro tubes at a final volume of $25 \mu$ containing $25 \mathrm{mM}$ HEPES, $\mathrm{pH}$ 7.5, $100 \mu \mathrm{M}$ Cys (Calbiochem), $50 \mu \mathrm{M}$ GlcN-Ins, prepared by cleavage of mycothiol bimane with mycothiol-S-conjugate amidase [19], $1 \mathrm{mM}$ ATP (Sigma), $1 \mathrm{mM} \mathrm{MgCl} 2,1 \mathrm{mM}$ DTT (ICN Biomedicals), and $12 \mu \mathrm{l}$ of different dilutions of protein extracts or affinity-purified fractions. The reaction mixture was incubated at $37^{\circ} \mathrm{C}$ for $30 \mathrm{~min}$. After incubation, thiols were derivatized by addition of $25 \mu 1$ of $8 \mathrm{mM}$ monobromobimane (Sigma) in acetonitrile and incubated at $60^{\circ} \mathrm{C}$ for 10 additional minutes. Finally, reactions were quenched by addition of $50 \mu \mathrm{l}$ of cold $20 \mathrm{mM}$ methanesulfonic acid. The resulting mixture $(5-10 \mu 1)$ was analyzed by LCMS using a Waters Symmetry C18 $5 \mu \mathrm{M}$ column $(4.6 \times 150 \mathrm{~mm})$ fitted with a QR $5 \mu \mathrm{m}$ C18 cartridge $(4.6 \mathrm{~mm} \times 1 \mathrm{~cm})$. The gradient elution using $5 \mathrm{mM}$ ammonium formate, $\mathrm{pH} 6.5$, and $\mathrm{MeOH}$ consisted of four steps at a flow rate of $0.5 \mathrm{ml} \mathrm{min}{ }^{-1}: 5-12 \% \mathrm{MeOH}$ in $4 \mathrm{~min}, 12-25 \% \mathrm{MeOH}$ in $6 \mathrm{~min}, 25-100 \%$ in $5 \mathrm{~min}$, followed by a $3-$ min wash with $100 \%$ methanol. Detection of derivatized thiols was accomplished using a fluorescence detector with excitation at $398 \mathrm{~nm}$ and emission at $480 \mathrm{~nm}$. Under these chromatographic conditions Cys-GlcN-Ins bimane showed a retention time of $14.5 \mathrm{~min}(\mathrm{~m} / \mathrm{z} 635,657,673)$.

\section{Results and discussion}

\section{Construction of MshC expression plasmids}

The production of recombinant $\mathrm{MshC}$ is a major prerequisite for the screening of potential mycothiol ligase inhibi- 
tors. However, the isolation of mycothiol ligase from $M$. smegmatis proved to be an arduous task [6] such that isolation of sufficient quantities of active enzyme from $M$. smegmatis would be impractical. We therefore sought to generate a strain that expresses significant amounts of soluble and active recombinant mycothiol ligase that is amenable to affinity purification. Expression of a target protein fused with another protein of intrinsically high solubility is a well known strategy to produce soluble recombinant proteins [20-22]. We began these efforts by first cloning the MshC gene (Rv2130c, $1245 \mathrm{bp}$ ) from M. tuberculosis into pGEV2 [15], pET42a and pMALc2X plasmids under the control of $\mathrm{T} 7$ or tac promoters to generate respective fusion proteins GB1-MshC, GST-MshC and MBP-MshC for expression in E. coli. However, despite enhanced expression in E. coli relative to the $\mathrm{MshC}$ gene on its own, all fusion proteins were produced primarily as insoluble inclusion bodies. Attempts to improve expression and solubility of these proteins, including use of codon optimized E. coli strains and induction with varying amounts of isopropyl $\beta$ D-thiogalactoside (IPTG) at different cell densities alone or in combination with varying temperatures, were unsuccessful.

Recombinant mycobacterial proteins have proved notoriously difficult to express in E. coli [9-12]. Mycobacteria have a high GC content (65.6\% in M. tuberculosis) and unique codon preferences, and the encoded proteins tend to be rich in glycine, alanine, proline and arginine, all of which can be detrimental for overexpression in $E$. coli [9]. Since $E$. coli was not found to be a suitable host for the production of recombinant MshC, we investigated the possibility of producing the same recombinant fusion proteins in the fast growing non-pathogenic bacterium $M$. smegmatis. For this study we selected the I64 M. smegmatis mutant as a host strain for expression of these MshC fusion proteins. Because this mutant lacks MshC activity and is deficient in mycothiol production [4], we foresaw the potential of using mycothiol production as an indicator of our constructs' activity. To generate mycobacterial compatible expression vectors, the constructs generated for expression of tagged MshC in E. coli, namely pGEV/MshC (GB1-MshC), pET42a/MshC-2 (GST-MshC) and pMALc2X/MshC (MBP-MshC), were next employed as templates for the amplification of the fragments to be cloned into the mycobacterial expression vector pACE (8191 bp) $[13,14]$. The set of expression vectors were generated by either inserting a PCR product of MshC with its N-terminal tag into pACE or by inserting the $\mathrm{N}$-terminal tag alone into $\mathrm{pACE} / \mathrm{MshC}$. The first strategy proved successful in the construction of the GST-MshC and MBP-MshC fusion proteins while for GB1-MshC it was necessary to insert the GB1 fragment into the $\mathrm{pACE} / \mathrm{MshC}$ plasmid.

In all cases, MshC was first cloned into the two unique cloning sites BamHI/ClaI of pACE. Once the plasmid pACE/MshC (9412 bp) was obtained, two additional unique cloning sites, NdeI and SpeI, were introduced by two separate site directed mutagenesis reactions. The result- ing vector named $\mathrm{pACE} / \mathrm{MshC}-2$ (9424 bp) was then digested with $N d e \mathrm{I}$ and $\mathrm{ClaI}$ (for GST-MshC and MBPMshC) and with NdeI/BamHI (for introduction of GB1). It is important to note that the MshC gene also contains a ClaI restriction site at position 1217. For purposes of keeping the MshC gene intact, the GST-MshC and MBP-MshC PCR products were generated with an $A c l$ I restriction site which is compatible with $C l a \mathrm{I}$. After cloning into this site it is converted into a non-cleavable Acll/ClaI hybrid. However, when pACE/MshC is digested with ClaI, there remains a possibility that the restriction enzyme will cut MshC within the gene at position 1217. Consequently, even after gel purification, it is possible that some of the linear vector may contain a small fragment of the $\mathrm{MshC}$ gene corresponding to base pairs 1217-1245. Sequencing of the GST-MshC and MBP-MshC expression plasmids showed that neither contains the additional fragment. The resulting expression vectors were identified as pACE/GB1MshC (9595 bp), pACE/GSTMshC (10246 bp) and pACE/ MBPMshC (10588 bp) (Fig. 2).

\section{Expression of tagged mycothiol ligase constructs in I64 M. smegmatis and evaluation of their ligase activity}

With the intent of improving protein expression and simplifying the purification of recombinant $\mathrm{MshC}$ (45.6 kDa), the MshC gene from M. tuberculosis (Rv2130c, $1245 \mathrm{bp}$ ) was fused at the N-terminus with GB1 (180 bp, $6.7 \mathrm{kDa}$ ), GST ( $837 \mathrm{bp}, 31.7 \mathrm{kDa}$, which includes C-terminal 6-His and S-tags) and MBP (1173 bp, $43.0 \mathrm{kDa})$ tags using the pACE vector that controls protein expression through the acetamidase promoter. pACE was previously generated by amplification of the acetamidase gene from M. smegmatis $[13,14]$. The acetamidase promoter consists of 4 ORFs identified as AmiS (642 bp), AmiD (285 bp), AmiA (429 bp) and $\mathrm{AmiC}(1074 \mathrm{bp})$ that are involved in regulation and transport. The acetamidase from $M$. smegmatis is an inducible enzyme and enables the organism to use several amides, such as acetamide as sole carbon sources $[23,24]$. Upon addition of $0.4 \%$ acetamide to the medium, the three fusion proteins were expressed at maximum levels after $22-26 \mathrm{~h}$ of incubation and were present in the soluble fraction. Analysis of the resulting protein extracts on SDS-PAGE indicated that the three fusion proteins migrated at the expected molecular weight and that the fusion tags are well tolerated in the I64 M. smegmatis mutant (Fig. 3). Expected molecular weights of GB1-MshC, GST-MshC and MBPMshC fusion proteins are $52.4 \mathrm{kDa}, 77.3 \mathrm{kDa}$ and $88.7 \mathrm{kDa}$, respectively.

In addition to the fusion proteins, it appears that upon induction with acetamide, each of the three transformed strains expresses two other major proteins migrating at $\sim 45 \mathrm{kDa}$ and $\sim 60 \mathrm{kDa}$ (Fig. 3, lanes 2-6). The band at $\sim 45 \mathrm{kDa}$ may represent acetamidase, whose molecular weight is calculated to be $43 \mathrm{kDa}$, as there is precedent for levels of this enzyme increasing up to 100 -fold in the presence of suitable substrate such as acetamide $[23,24]$. In the 


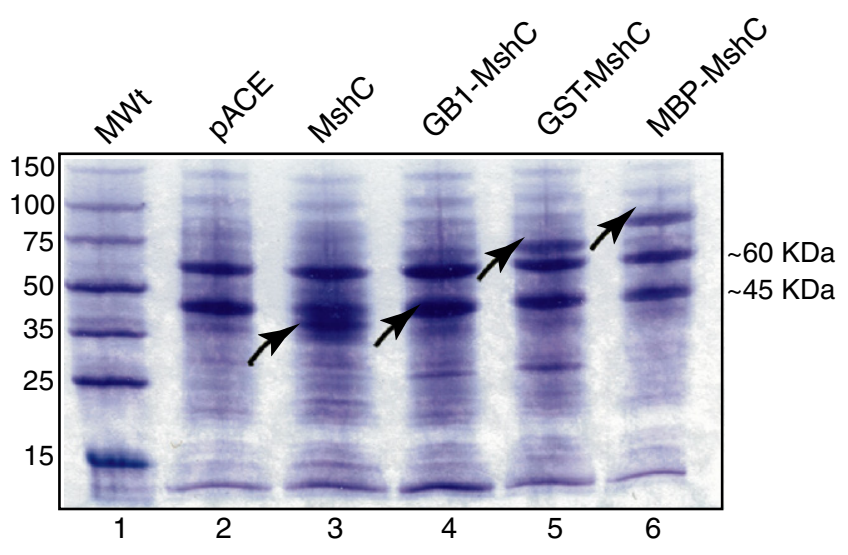

Fig. 3. SDS-PAGE analysis of protein extracts. Lanes 1-6 contain a molecular weight marker and protein extracts of I64pACE, I64pACE/ MshC, I64pACE/GB1MshC, I64pACE/GSTMshC and I64pACE/ MBPMshC, respectively. Molecular weights in $\mathrm{kDa}$ are labeled to the left of lane 1 and acetamidase-induced MshC constructs are marked with arrows. The strong bands at approximately 45 and $60 \mathrm{kDa}$ that are present in lanes 2-6 appear upon induction with acetamide.

Table 2

Specific activity of protein extracts and purified fractions obtained from selected strains in expression medium ${ }^{\mathrm{a}}$

\begin{tabular}{ll}
\hline Strain/construct & $\begin{array}{l}\text { Specific activity } \\
\left(\mathrm{nmol} \mathrm{min} \mathrm{mg}^{-1}\right)\end{array}$ \\
\hline M. smegmatis $\mathrm{mc}^{2} 155$ & 0.06 \\
M. smegmatis I64pACE & nd \\
M. smegmatis I64pACE/MshC & 0.2 \\
M. smegmatis I64pACE/GB1-MshC & 0.1 \\
M. smegmatis I64pACE/GST-MshC & 0.3 \\
M. smegmatis I64pACE/MBP-MshC & 0.3 \\
\hline
\end{tabular}

${ }^{a}$ Specific activity of supernatants is based on total protein determined by the Bradford protein assay.

case of the GB1-MshC construct, this fusion protein migrated together with the band of $45 \mathrm{kDa}$ (Fig. 3, lane 4). However, expression of GB1-MshC was unambiguously demonstrated by measurement of its ligase activity (Table 2) and by complementation of the mycothiol deficient I64 mutant (Fig. 4). Ligase activity for all protein extracts is summarized in Table 2. All three of the fusion proteins showed ligase activity and the highest levels were observed for GST-MshC and MBP-MshC, both of which exhibited specific activities of $0.3 \mathrm{nmol} \mathrm{min}{ }^{-1} \mathrm{mg}^{-1}$. These results indicate that the fusion tags do not interfere with the activity of mycothiol ligase. However, if desired each of the fusion proteins can be cleaved within the linker separating MshC and its N-terminal tag. The GB1 and MBP fusion proteins contain thrombin and factor Xa cleavage sites, respectively, while the GST fused protein contains both.

\section{Complementation of the mycothiol deficient I64 M. smegmatis mutant}

The I64 M. smegmatis mutant used in this study was generated previously by chemical mutagenesis of $M$. smegmatis $\mathrm{mc}^{2} 155$. The I64 mutant strain was shown to be deficient in mycothiol production producing only $1 \%$ of the
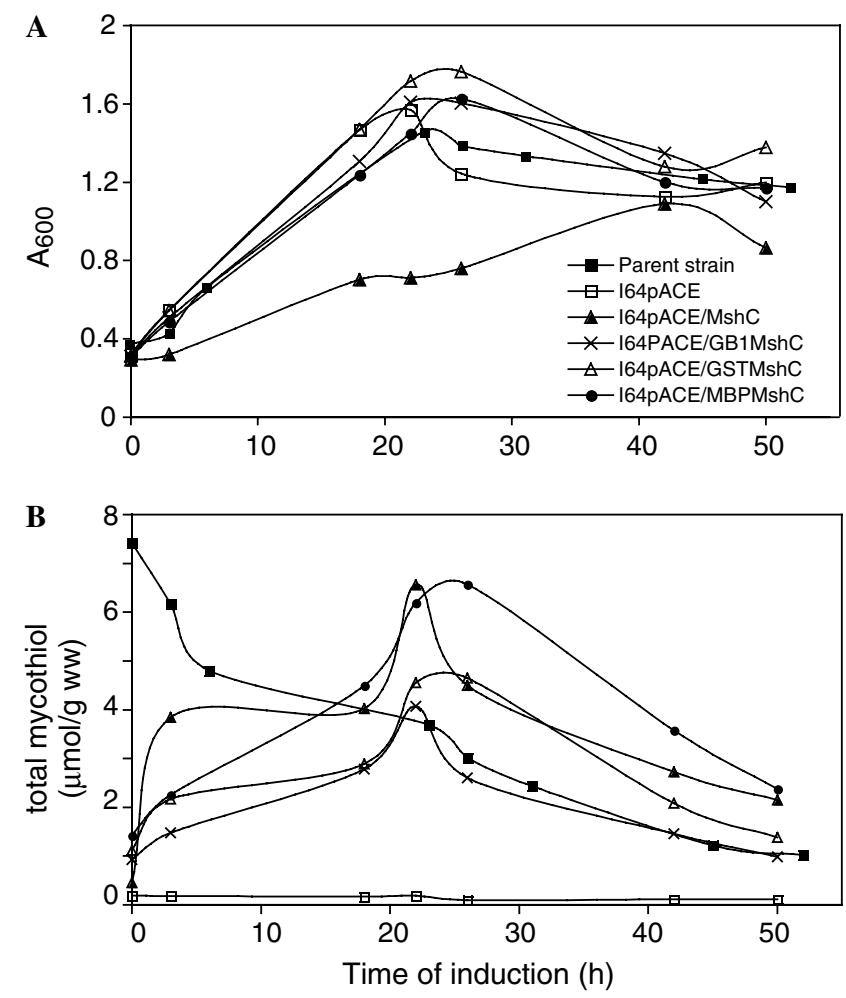

Fig. 4. Complementation of the mycothiol deficient I64 M. smegmatis mutant. (A) Growth rate measured as cell density $\left(A_{600}\right)$ versus time (h). (B) Total mycothiol content of wild type $\mathrm{mc}^{2} 155$ and I64 M. smegmatis recombinant strains after transfer to expression medium and induction with acetamide. Starter cultures of all strains were grown in nutrient-rich $7 \mathrm{H}$ /OADC/Tween prior to inoculating a larger volume of expression medium.

amount of mycothiol as its parent strain $(0.1 \mu \mathrm{mol} / \mathrm{g}$ versus $10 \mu \mathrm{mol} / \mathrm{g}$ dry weight, respectively), and I64 M. smegmatis exhibits increased sensitivity to free radicals, alkylating agents and a variety of antibiotics. MshC activity in this mutant strain was shown to be $\leqslant 2 \%$ that of its parent strain. Sequencing of the $m s h C$ gene revealed that a single amino acid substitution, Leu 205 to Pro, is responsible for the reduced mycothiol production and its associated phenotype [4]. The I64 M. smegmatis strain was thus chosen for expression of recombinant $M$. tuberculosis MshC to prevent any interference from endogenous wild type $M$. smegmatis $\mathrm{MshC}$ and to facilitate complementation experiments.

To determine whether the recombinant MshC fusion proteins were active, I64 pACE/GB1-MshC, I64 pACE/ GST-MshC, and I64 pACE/MBP-MshC were transformed into I64 M. smegmatis and acetamidase-induced mycothiol ligase activity and mycothiol production of these recombinant strains was measured in parallel with those of the I64 $M$. smegmatis mutant (I64pACE), its parent strain $M$. smegmatis $\mathrm{mc}^{2} 155$, and non-tagged MshC (I64 pACE/ $\mathrm{MshC}$ ). The levels of mycothiol of all the strains were determined at multiple stages of growth (up to $55 \mathrm{~h}$ ) in expression medium under antibiotic pressure, except for the parent strain $\mathrm{mc}^{2} 155$ which was grown in the absence of 
antibiotics. The I64pACE strain produced on average around $0.1 \mu \mathrm{mol}$ of total mycothiol per gram wet weight, averaging less than $5 \%$ of the levels produced by the parent strain during growth (Fig. 4). In general, all strains containing constructs of tagged and non-tagged MshC produced mycothiol levels between 2 and 69 times higher (depending on time from induction) than the I64pACE mutant, or levels at least as great as those of the parent strain grown under identical conditions (Fig. 4B). The three different recombinant strains had a maximum peak of mycothiol production at $22-26 \mathrm{~h}$ after induction. After this point, the levels of mycothiol decline in parallel with the growth curve, most probably due to the consumption of the acetamide used as a source of nitrogen and carbon (Fig. 4A). These results indicated that the three fusion proteins are functional in vivo and complement the ligase activity in the I64 M. smegmatis mutant. Of the three recombinant strains, I64pACE/MBP-MshC produced the highest levels of mycothiol, namely $6.6 \mu \mathrm{mol} / \mathrm{g}$ wet weight at $26 \mathrm{~h}$, which is a $2.2-$ fold increase relative to the parent strain.

\section{Purification of $r G S T-M$ sh C and $r M B P-M \operatorname{sh} C$}

On the basis of the observed complementation of the mycothiol deficient I64 M. smegmatis mutant by the three constructs (Fig. 4) and evaluation of their ligase activity (Table 2), I64pACE/GST-MshC and I64pACE/MBP$\mathrm{MshC}$ were selected for affinity purification of recombinant protein. These recombinant strains were grown on a $2-\mathrm{L}$ scale and processed as described in "Materials and methods" section. The entire protocol for expression and purification required 5 days for growth and induction and approximately $8 \mathrm{~h}$ to generate cleared cell lysates and to purify the proteins by affinity chromatography on glutathione or amylose resin columns. As seen in the SDS-PAGE denaturing gels in Fig. 5, affinity purifications of the GSTand MBP-tagged MshC proteins were very effective and yielded fractions containing fusion proteins of the expected molecular weights $M_{\mathrm{r}} \sim 77$ and $\sim 88 \mathrm{kDa}$ that were at least $90 \%$ pure (as determined by SDS-PAGE and LC-MS). Starting from a 2-L culture of $M$. smegmatis I64pACE/ GST-MshC or I64pACE/MBP-MshC, $0.7 \mathrm{mg} / \mathrm{L}$ of pure rGST-MshC and $1.2 \mathrm{mg} / \mathrm{L}$ of pure rMBP-MshC were obtained (Table 3). The molecular weights of the affinitypurified GST-MshC and MBP-MshC fractions were confirmed by LC-MS giving observed masses of $77.28 \mathrm{kDa}$ and

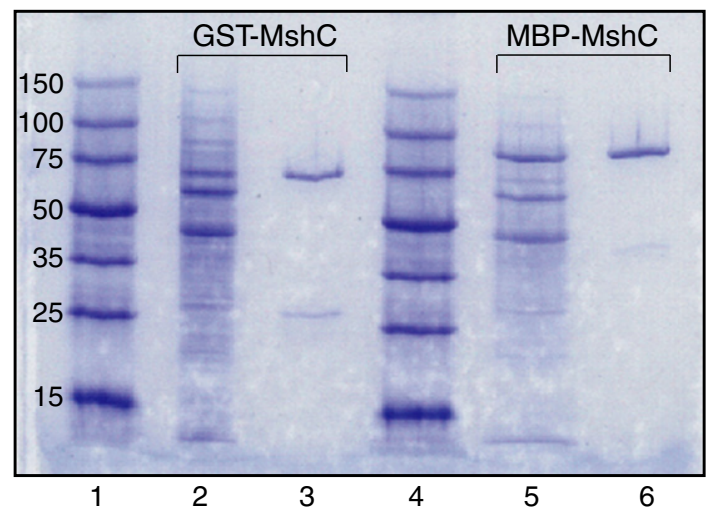

Fig. 5. SDS-PAGE analysis of protein extracts and affinity-purified recombinant GST-MshC and MBP-MshC. Lanes 1 and 4, molecular weight markers $(\mathrm{kDa})$; lanes 2 and 5, protein extracts of I64pACE/GSTMshC and I64pACE/MBPMshC; lanes 3 and 6, affinity-purified GSTMshC and MBP-MshC.

$88.68 \mathrm{kDa}$, respectively, in excellent agreement with the calculated molecular weights of 77,275.8 $\mathrm{Da}$ and 88,679.1 Da. Evident from the SDS-PAGE and detectable by LC-MS analysis, two minor species with respective masses of 31.8 and $42.9 \mathrm{kDa}$ are observed in the GST-MshC and MBP$\mathrm{MshC}$ affinity-purified fractions (Fig. 5, lanes 3 and 6). As the predicted masses of GST and MBP are 31.8 and $42.7 \mathrm{kDa}$ respectively, these impurities likely correspond to the GST and MBP tags as a result of proteolysis within the spacer separating the tags and MshC.

Mycothiol ligase activities of total protein extracts and combined affinity-purified fractions for rGST-MshC and rMBP-MshC were measured using the protocol described in "Materials and methods" section and yielded final specific activities of $9 \mathrm{nmol} \mathrm{min}^{-1} \mathrm{mg}^{-1}$ for purified rGST-MshC and $25 \mathrm{nmol} \mathrm{min}^{-1} \mathrm{mg}^{-1}$ for purified rMBP-MshC. Because the greatest recovery and specific activity was observed with rMBP-MshC (Table 3), this construct will likely be chosen for larger scale protein production and used in screening efforts aimed at identifying small molecule inhibitors of MshC. In anticipation of such efforts, we have monitored at approximately two-week intervals the activity of rMBPMshC stored as $20 \%$ glycerol stocks at $-80^{\circ} \mathrm{C}$. Thus far, we have observed no detectable loss of activity upon storage under these conditions for at least three months (data not shown), further indicating that these constructs and protocols will allow for production of active enzyme in quantities sufficient to support future studies.

Table 3

Purification of recombinant GST-MshC and MBP-MshC proteins ${ }^{\mathrm{a}}$

\begin{tabular}{lccc}
\hline Step & Total protein $(\mathrm{mg})$ & Total activity $\left(\mathrm{nmol} \mathrm{min}{ }^{-1}\right)$ & Specific activity $\left(\mathrm{nmol} \mathrm{min}^{-1} \mathrm{mg}^{-1}\right)$ \\
\hline Total protein extract & & & \\
GST-MshC & 118.1 & 36.5 & 0.3 \\
rGST-MshC & 0.7 & 6.3 & 9.0 \\
MBP-MshC & 82.7 & 26.9 & 0.3 \\
rMBP-MshC & 1.2 & 22.3 & 25.0 \\
\hline
\end{tabular}

\footnotetext{
${ }^{\text {a }}$ Specific activity of supernatants is based on total protein determined by the Bradford protein assay.
} 


\section{Concluding remarks}

In summary, we have demonstrated that recombinant MshC fusion proteins can be expressed in M. smegmatis using the pACE vector under the control of the acetamidase promoter. The GB1, GST and MBP tags are well tolerated in the I64 M. smegmatis mutant and the resulting MshC fusion proteins are functional in vivo. Acetamidaseinduced expression of each of these constructs greatly improved the yield of $\mathrm{MshC}$ relative to the naturally occurring levels produced by the wild type strain $M$. smegmatis $\mathrm{mc}^{2} 155$. The yields obtained with this system for the GST$\mathrm{MshC}$ and MBP-MshC protein ranged from 0.7 to $1.2 \mathrm{mg}$ of pure and active protein/L. The specific mycothiol ligase activities measured for rGSTMshC and rMBPMshC were $9 \mathrm{nmol} \mathrm{min}{ }^{-1} \mathrm{mg}^{-1}$ and $25 \mathrm{nmol} \mathrm{min}{ }^{-1} \mathrm{mg}^{-1}$, respectively. In particular, recombinant GST- and MBP-tagged MshC constructs can be produced at levels that will support screening efforts aimed at identifying inhibitors of mycothiol ligase. If desired, MshC may also be cleaved from its N-terminal tag by incubation with proteases Factor Xa (MBP-MshC and GST-MshC) or thrombin (GST-MshC) with negligible losses in quantity or activity (M.T.G.L. and C.A.B., unpublished data). This expression system represents a robust method for expression of recombinant mycobacterial proteins that cannot be expressed in other heterologous systems such as E. coli.

\section{Acknowledgments}

This work was supported in part by the Intramural Research Program, NIH (National Institute of Diabetes and Digestive and Kidney Diseases), by the Intramural AIDS Targeted Antiviral Program of the Office Director, NIH (C.A.B.) and grant AI49174 from the National Institute of Allergy and Infectious Diseases, NIH (R.C.F.).

\section{References}

[1] G.L. Newton, K. Arnold, M.S. Price, C. Sherrill, S.B. Delcardayre, Y. Aharonowitz, G. Cohen, J. Davies, R.C. Fahey, C. Davis, Distribution of thiols in microorganisms: mycothiol is a major thiol in most actinomycetes, J. Bacteriol. 178 (1996) 1990-1995.

[2] G.L. Newton, R.C. Fahey, Mycothiol biochemistry, Arch. Microbiol. 178 (2002) 388-394.

[3] N.A. Buchmeier, G.L. Newton, T. Koledin, R.C. Fahey, Association of mycothiol with protection of Mycobacterium tuberculosis from toxic oxidants and antibiotics, Mol. Microbiol. 47 (2003) 1723-1732.

[4] M. Rawat, G.L. Newton, M. Ko, G.J. Martinez, R.C. Fahey, Y. AvGay, Mycothiol-deficient Mycobacterium smegmatis mutants are hypersensitive to alkylating agents, free radicals, and antibiotics, Antimicrob. Agents Chemother. 46 (2002) 3348-3355.

[5] G.M. Nicholas, C.A. Bewley, Inhibitors of mycothiol-S-conjugate amidase and related genes, Curr. Med. Chem.-Anti-Infective Agents 3 (2004) 221-231.

[6] D. Sareen, M. Steffek, G.L. Newton, R.C. Fahey, ATP-dependent Lcysteine:1D-myo-inosityl2-amino-2-deoxy- $\alpha$-D-glucopyranoside ligase, mycothiol biosynthesis enzyme MshC, is related to class I cysteinyl-tRNA synthetases, Biochemistry 41 (2002) 6885-6890.

[7] D. Sareen, G.L. Newton, R.C. Fahey, N.A. Buchmeier, Mycothiol is essential for growth of Mycobacterium tuberculosis Erdman, J. Bacteriol. 185 (2003) 6736-6740.

[8] C.M. Sassetti, D.H. Boyd, E.J. Rubin, Genes required for mycobacterial growth defined by high density mutagenesis, Mol. Microbiol. 48 (2003) 77-84.

[9] S. Daugelat, J. Kowall, J. Mattow, D. Bumann, R. Winter, R. Hurwitz, S.H.E. Kaufmann, The RD1 proteins of Mycobacterium tuberculosis: expression in Mycobacterium smegmatis and biochemical characterization, Microb. Infect. 5 (2003) 1082-1095.

[10] R. Vincentelli, S. Canaan, V. Campanacci, C. Valencia, D. Maurin, F. Frassinetti, L. Scappucini-Calvo, Y. Bourne, C. Cambillau, C. Bignon, High-throughput automated refolding screening of inclusion bodies, Protein Sci. 13 (2004) 2782-2792.

[11] A. Korepanova, F.P. Gao, Y. Hua, H. Qin, R.K. Nakamoto, T.A. Cross, Cloning and expression of multiple integral membrane proteins from Mycobacterium tuberculosis in Escherichia coli, Protein Sci. 14 (2005) 148-158.

[12] S. Ahmad, M.M. Ali, A.S. Mustafa, Construction of a modified vector for efficient purification of recombinant Mycobacterium tuberculosis proteins expressed in Escherichia coli, Protein Expres. Purif. 29 (2003) $167-175$.

[13] K.A.L. De Smet, K.E. Kempsell, A. Gallagher, K. Duncan, D.B. Young, Alteration of a single amino acid residue reverses fosfomycin resistance of recombinant MurA from Mycobacterium tuberculosis, Microbiology 145 (1999) 3177-3184.

[14] T. Parish, E. Mahenthitalingam, P. Draper, E.O. Davis, M.J. Colston, Regulation of the inducible acetamidase gene of Mycobacterium smegmatis, Microbiology 143 (1997) 2267-2276.

[15] J.F. Huth, C.A. Bewley, B.M. Jackson, A.G. Hinnebusch, G.M. Clore, A.M. Gronenborn, Design of an expression system for detecting folded protein domains and mapping macromolecular interactions by NMR, Protein Sci. 6 (1997) 2359-2364

[16] J. Sambrook, E.F. Fritsch, T. Maniatis, Commonly used techniques in molecular cloning, in: second ed., Molecular Cloning A Laboratory Manual, vol. 3, Cold Spring Harbor Laboratory Press, Cold Spring Harbor, NY, 1989, pp. E10-E14.

[17] M.L. Beggs, J.T. Crawford, K.D. Eisenach, Isolation and sequencing of the replication region of Mycobacterium avium plasmid pLR7, J. Bacteriol. 177 (1995) 4836-4840.

[18] C.G. Stanley, P.H. von Hippel, Calculation of protein extinction coefficients from amino acid sequence data, Anal. Chem. 182 (1989) 319-326.

[19] G.L. Newton, Y. Av-Gay, R.C. Fahey, A novel mycothiol-dependent detoxification pathway in Mycobacteria involving mycothiol $S$-conjugate amidase, Biochemistry 39 (2000) 10739-10746.

[20] Y-P. Shih, W.-M. Kung, J.-C. Chen, C.-H. Yeh, A.H.-J. Wang, T.-F. Wang, High throughput screening of soluble proteins, Protein Sci. 11 (2002) 1714-1719.

[21] P. Braun, Y. Hu, B. Shen, A. Halleck, M. Koundinya, E. Harlow, J. LaBaer, Proteome-scale purification of human proteins from bacteria, Proc. Natl. Acad. Sci. USA 99 (2002) 2654-2656.

[22] M. Hammarström, N. Hellgren, S. Van Den Berg, H. Berglund, T. Härd, Rapid screening for improved solubility of small proteins produced as fusion proteins in Escherichia coli, Protein Sci. 11 (2002) 313-321.

[23] E. Mahenthiralingam, P. Draper, E.O. Davis, M.J. Colston, Cloning and sequencing of the gene which encodes the highly inducible acetamidase from Mycobacterium smegmatis, J. Gen. Microbiol. (1993) 575-583.

[24] G. Roberts, D.G.N. Muttucumaru, T. Parish, Control of the acetamidase gene of Mycobacterium smegmatis by multiple regulators, FEMS Microbiol. Lett. 221 (2003) 131-136. 